\title{
Probing the Neural Mechanisms for Distractor Filtering and Their History-Contingent Modulation by Means of TMS
}

\author{
Carlotta Lega, ${ }^{1}$ Oscar Ferrante, ${ }^{1}$ Francesco Marini, ${ }^{2}$ Elisa Santandrea, ${ }^{1}{ }^{-}$Luigi Cattaneo, ${ }^{1,3}$ and ${ }^{\circ}$ Leonardo Chelazzi ${ }^{1,3}$ \\ ${ }^{1}$ Department of Neuroscience, Biomedicine and Movement Sciences, University of Verona, Verona I-37134, Italy, ${ }^{2}$ Swartz Center for Computational \\ Neuroscience, University of California San Diego, La Jolla CA 92093, California, and ${ }^{3}$ Italian Institute of Neuroscience, Verona I-37134, Italy
}

In visual search, the presence of a salient, yet task-irrelevant, distractor in the stimulus array interferes with target selection and slows down performance. Neuroimaging data point to a key role of the frontoparietal dorsal attention network in dealing with visual distractors; however, the respective roles of different nodes within the network and their hemispheric specialization are still unresolved. Here, we used transcranial magnetic stimulation (TMS) to evaluate the causal role of two key regions of the dorsal attention network in resisting attentional capture by a salient singleton distractor: the frontal eye field (FEF) and the cortex within the intraparietal sulcus (IPS). The task of the participants (male/female human volunteers) was to discriminate the pointing direction of a target arrow while ignoring a task-irrelevant salient distractor. Immediately after stimulus onset, triple-pulse $10 \mathrm{~Hz}$ TMS was delivered either to IPS or FEF on either side of the brain. Results indicated that TMS over the right FEF significantly reduced the behavioral cost engendered by the salient distractor relative to left FEF stimulation. No such effect was obtained with stimulation of IPS on either side of brain. Interestingly, this FEF-dependent reduction in distractor interference interacted with the contingent trial history, being maximal when no distractor was present on the previous trial relative to when there was one. Our results provide direct causal evidence that the right FEF houses key mechanisms for distractor filtering, pointing to a pivotal role of the frontal cortex of the right hemisphere in limiting interference from an irrelevant but attention-grabbing stimulus.

Key words: distractor filtering; dorsal attention network; frontal eye field; intraparietal sulcus; transcranial magnetic stimulation

\section{Significance Statement}

Visually conspicuous stimuli attract our attention automatically and interfere with performance by diverting resources away from the main task. Here, we applied transcranial magnetic stimulation over four frontoparietal cortex locations (frontal eye field and intraparietal sulcus in each hemisphere) to identify regions of the dorsal attention network that help limit interference from task-irrelevant, salient distractors. Results indicate that the right FEF participates in distractor-filtering mechanisms that are recruited when a distracting stimulus is encountered. Moreover, right FEF implements adjustments in distraction-filtering mechanisms following recent encounters with distractors. Together, these findings indicate a different hemispheric contribution of the left versus right dorsal frontal cortex to distraction filtering. This study expands our understanding of how our brains select relevant targets in the face of task-irrelevant, salient distractors.

\section{Introduction}

Visual selective attention supports an individual's ability to select relevant information while disregarding irrelevant but attentiongrabbing stimuli (Jonides and Yantis, 1988; Yantis and Jonides,

Received Oct. 23, 2018; revised July 19, 2019; accepted July 22, 2019.

Author contributions: C.L. and 0.F. performed research; C.L., O.F., F.M., E.S., L. Cattaneo, and L. Chelazzi analyzed data; C.L. wrote the first draft of the paper; C.L., 0.F., F.M., E.S., L. Cattaneo, and L. Chelazzi edited the paper; C.L., F.M., E.S., and L. Chelazzi wrote the paper; L. Chelazzi designed research.

This work was supported by University of Verona Ricerca di Base 2015 (Grant B32F15000700001) to L. Chelazzi.

The authors declare no competing financial interests.

Correspondence should be addressed to Leonardo Chelazzi at leonardo.chelazzi@univr.it.

https://doi.org/10.1523/JNEUROSCI.2740-18.2019

Copyright $@ 2019$ the authors
1990; Forster and Lavie, 2008; Marini et al., 2013). Evidence that attention is involuntarily captured by salient stimuli comes, among others, from studies using visual search arrays with salient singleton distractors, such as a uniquely colored stimulus in an array of differently colored items (Theeuwes, 1992; Theeuwes and Burger, 1998; Theeuwes and Godljn, 2002). The presence of a singleton distractor in a search array results in an unwanted shift of attention to the salient stimulus (attentional capture effect), as indexed by a measurable performance cost relative to trials without the singleton distractor. Stimulus-driven mechanisms responsible for the attentional capture effects are antagonized by goal-driven mechanisms that guide attention toward the relevant target stimulus and suppress distractors (Reynolds and Chelazzi, 
2004; Müller and Ebeling, 2008; Chelazzi et al., 2011; Marini et al., 2016), although the cognitive and neural mechanisms that support distractor filtering are still under debate.

Distractor filtering, which consists of the elimination, or at least the attenuation, of the behavioral costs engendered by irrelevant yet salient stimuli, has received mounting interest in recent years (Chelazzi et al., 2019). Different putative mechanisms for distractor filtering have been proposed depending on the adopted experimental paradigm and behavioral context (Chelazzi et al., 2019). These mechanisms include proactive control (Marini et al., 2013, 2016; Geng, 2014; Cosman et al., 2018), habituation of capture (Neo and Chua, 2006; Pascucci and Turatto, 2015; Turatto et al., 2018a,b), intertrial priming (Geyer et al., 2008; Müller et al., 2010), and implicit distractor probability learning (Goschy et al., 2014; Ferrante et al., 2018; Wang and Theeuwes, 2018a,b; Sauter et al., 2018, 2019; Di Caro et al., 2019). Despite the abundance of behavioral evidence about specific task conditions and behavioral contexts wherein distractor suppression occurs, much less is known about the underlying neural mechanisms (Egeth et al., 2010; Folk and Remington, 2010; Seidl et al., 2012; Gaspar and McDonald, 2014; Geng, 2014; Liesefeld et al., 2014, 2017; Marini et al., 2016; Donohue et al., 2018). Therefore, the present study sought to characterize the neural machinery for distractor filtering by investigating the causal role of different subregions within the dorsal attention network.

Numerous functional imaging studies demonstrated that attentional control in the presence of potential distraction is supported by the dorsal (mostly bilateral) frontoparietal attention network, whose core regions include the frontal eye field (FEF) and the posterior parietal cortex (PPC), notably tissue within the intraparietal sulcus (IPS) (Corbetta and Shulman, 2002; de Fockert et al., 2004; Serences et al., 2004, 2005; Leber, 2010; Talsma et al., 2010; de Fockert and Theeuwes, 2012; DiQuattro et al., 2014; Marini et al., 2016; Lee and Geng, 2017). These anatomical locations align well with electrophysiological evidence in nonhuman primates, showing that both target selection and distractor suppression mechanisms involve parietal cortex (Ipata et al., 2006) and PFC (for a specific contribution of FEF in distractor suppression, see Cosman et al., 2018), although neural responses to salient distractors are more greatly suppressed and more closely correlated with performance in prefrontal, rather than parietal cortex (Suzuki and Gottlieb, 2013).

Brain imaging studies in humans also reported a correlation between frontal (but not parietal) neural activity and the magnitude of distractor interference, strongly supporting a role for the frontal cortex in actively preventing the interference from irrelevant distractors (de Fockert et al., 2004; de Fockert and Theeuwes, 2012; Marini et al., 2016). Consistently, event-related potential (ERP) studies also converge in indicating a prominent role of frontal areas in attentional control (Grent-'t-Jong and Woldorff, 2007; Leblanc et al., 2008; Brignani et al., 2009; Ptak et al., 2011; Shomstein et al., 2012; Liesefeld et al., 2014). These findings also revealed an orderly temporal structure of neural signatures during the control of spatial attentional allocation, with attentional control signals first elicited at the level of the frontal lobe, followed by activity in the parietal lobe. This temporal advantage for the frontal over the parietal signals likely reflects processes involved in attentional deployment, but also in target selection and conflict control. Together, these results seem to indicate a leading contribution of the frontal areas in monitoring (potential) conflict and proactively preventing or reactively abating distraction. However, an inherent limitation of these studies is the inability to reveal any causal organization in the described relationship between brain activity and behavioral performance. To probe causal relationships between brain and behavior, noninvasive brain stimulation techniques are the most suitable choice (Pascual-Leone et al., 2000). Cosman et al. (2015) demonstrated that anodal transcranial direct-current stimulation (tDCS) over PFC enhanced the ability of participants to overcome distraction by decreasing the reaction time (RT) cost associated with a salient but task-irrelevant item. Along the same lines, by using repetitive transcranial magnetic stimulation (TMS) over PPC, Hodsoll et al. (2009) found that TMS over the right PPC, but not the left PPC, significantly reduced the RT cost of distraction. Moreover, the frontoparietal network is causally involved in filtering out not only perceptually salient, but also conflicting distractors, as demonstrated by modulations in the interference elicited by task-irrelevant flanker stimuli after tDCS of either PFC (Zmigrod et al., 2016) or parietal cortex (Weiss and Lavidor, 2012; Kajimura and Nomura, 2015).

Brain stimulation studies have revealed that the parietal and prefrontal cortices are involved in distractor filtering. However, due to the poor spatial resolution of tDCS and the lack of TMS or tDCS studies that directly compared the two crucial nodes of the dorsal frontoparietal network, namely, the FEF and IPS, it remains to be established whether distractor filtering is more distinctively supported by one or the other node of this network, or equally by both. Likewise, the available evidence is highly inconclusive as to whether the right and left dorsal attention networks are equally involved in distractor filtering. In the present study, we used TMS to comparatively investigate the causal role in distraction filtering of FEF and IPS on either side of the brain. Our general hypothesis was that the dorsal frontoparietal network is causally involved in distraction filtering, consistent with evidence in the literature (Chelazzi et al., 2019), but that this function might be more strongly supported by either node in the network. In particular, in light of previous neuroimaging observations (de Fockert et al., 2004; Melloni et al., 2012; de Fockert and Theeuwes, 2012; Marini et al., 2016) and the established temporal dynamics within the frontoparietal network, demonstrated by ERP studies (Grent-'t-Jong and Woldorff, 2007; Leblanc et al., 2008; Brignani et al., 2009; Ptak et al., 2011; Shomstein et al., 2012; Liesefeld et al., 2014), we might expect a more distinctive involvement of frontal regions in distractor suppression mechanisms, as indexed by a stronger modulation in the cost of distraction following FEF stimulation.

Cross-trial contingencies, such as the presence or absence of distractors in consecutive trials, are well known to modulate distraction filtering: for example, the RT cost engendered by a salient distractor is larger when a distractor was absent (vs present) in the immediately preceding trial (Geyer et al., 2008; Marini et al., 2013). A neuro-functional architecture for this effect may consist of an increase in top-down control following distractor encounters (Botvinick et al., 2001; Kerns et al., 2004), mediated by increased activity in the frontoparietal circuit (FEF and IPS, see Walsh et al., 2011). This view finds support in neurostimulation studies (Hodsoll et al., 2009; Soutschek et al., 2013) showing that the modulation of activity in the right PPC by means of TMS affected performance during conflict tasks, with the effect being strongly modulated as a function of what happened in the previous trial. Hodsoll et al. (2009) used an additional singleton paradigm to demonstrate that inhibitory $1 \mathrm{~Hz}$ TMS over the right PPC, but not left PPC, reduced the behavioral cost of a color-singleton distractor. Crucially, this lessening of distractor interference was mostly due to the elimination of 
priming effects between target and distractor singletons on consecutive trials. In this context, we therefore hypothesized an effect of TMS that varies depending on recent trial history, namely, depending on the state of the brain in the instant when it encounters a distractor. This idea also fits well with the notion of state dependency, whereby the effects of TMS on behavioral performance depend not only on the stimulation parameters themselves, but also on the brain's "state" when stimulation is applied (Silvanto and Pascual-Leone, 2008; Silvanto and Cattaneo, 2017). Based on this evidence, here we formulated the additional hypothesis that modulations of distraction filtering by cross-trial contingencies may likewise be mediated by the dorsal frontoparietal network, and therefore that FEF and/or IPS stimulation might alter such history-dependent modulations of distractor filtering. Therefore, if FEF and/or IPS are involved also in the regulation of cross-trial dynamics of distractor-filtering, we expected to identify an interaction between TMS stimulation on a given trial and the presence versus absence of a distractor in the preceding trial, a possibility that we addressed with the present work.

\section{Materials and Methods}

Participants. Thirty-two participants took part in the experiment (22 female; mean age $=23.56$ years, $\mathrm{SD}=3.44$ years). Two of them had to be excluded: 1 participant did not complete the task because FEF stimulation induced slight movements of the contralateral hand, which interfered with the task; another participant was excluded because of nearchance responses (mean accuracy: 58\%). Therefore, data from 30 participants (20 females, mean age $=23.4$ years, $S D=3.24$ years) were used for the analyses reported below. We would like to state candidly that, following the initial reviewing of our study, in compliance with the reviewers' recommendation, we increased the original sample size, from $N=20$ to the final $N=30$ subjects. To the benefit of complete transparency, the key statistical results on the original sample are available online (https://osf.io/4ke56/; stored on the Open Science Framework data sharing platform). All participants were right-handed and with normal or corrected-to-normal visual acuity and normal color vision. Before the TMS experiment, each subject filled out a questionnaire to evaluate eligibility for TMS. None of the participants reported any contraindications for TMS use (Rossi et al., 2009). Written informed consent was obtained from all participants before the beginning of the experiment. The study protocol was approved by the local ethical committee, and the experiment was conducted in accordance with the Declaration of Helsinki.

Materials and stimuli. The protocol was adapted from the additional singleton paradigm (Theeuwes, 1992). A version of the paradigm similar to the one used in the current experiment has been used previously in our laboratory (Ferrante et al., 2018). The visual search display consisted of four stimuli (one per visual quadrant) presented equidistantly from one another and centered on a central fixation point (eccentricity: $4^{\circ}$ ). All stimuli were composed of two green or red triangles $\left(1^{\circ} \times 1^{\circ}\right.$ each $)$ presented on a light gray background. In $50 \%$ of trials, all display items were of the same color (e.g., red; distractor-absent condition); whereas in the remaining $50 \%$ of trials, three items were of the same color (e.g., red) and the remaining item (additional singleton) was of the alternative color (e.g., green; distractor-present condition). The target was defined as the only item in the display with both triangles pointing in the same direction (both up or both down, i.e., a double arrowhead), whereas the singleton distractor, when present, was a color-singleton stimulus with both triangles pointing outwardly. The remaining stimuli (nontargets or fillers) were always of the same color as the target and with both triangles pointing inwardly (Fig. 1A).

Procedure. Participants were seated in front of a 17 inch CRT monitor (spatial resolution of $1280 \times 1024$ pixels and a refresh rate of $75 \mathrm{~Hz}$ ) at a distance of $57 \mathrm{~cm}$, in a dimly illuminated, silent room. A chin rest was used to keep the viewing distance constant during the whole session. Each trial began with a fixation point displayed in isolation for $1000 \mathrm{~ms}$, and this was then accompanied for $700 \mathrm{~ms}$ by an array of four placehold- ers, which were identical to nontargets. At the end of the $700 \mathrm{~ms}$ period, one of the placeholders was replaced by the target; and on $50 \%$ of the trials, a different placeholder was replaced by the singleton distractor (Tommasi et al., 2015; Ferrante et al., 2018). This array remained visible for $50 \mathrm{~ms}$ and was followed by a blank screen until the participant responded or $2000 \mathrm{~ms}$ had elapsed, whichever came first. The subsequent trial started after a $4000 \mathrm{~ms}$ intertrial interval (Fig. 1B). The participants' task was to indicate as quickly and as accurately as possible whether the target element was pointing up or down by pressing "1" for "up" or " 2 " for "down" on a numeric keypad (or vice versa; counterbalanced across participants). The experiment included two sessions conducted on 2 different days. In each session, participants completed a practice block of 24 trials to familiarize with the task, followed by 6 experimental blocks (one per TMS condition).

Each block consisted of 72 trials, 36 of which were distractor-present and 36 distractor-absent. Within each block, the target and the singleton distractor were presented equally often at any given spatial location. The order of blocks was pseudo-randomized in such a way that in the first session the six different TMS conditions were equally distributed across participants (to spread out any potential learning effect equally across TMS conditions). The second session was identical to the first one, except that the order of blocks was reversed relative to the first session (to minimize any carryover effects related to stimulation site). Each experimental session lasted $\sim 2 \mathrm{~h}$.

TMS. Online neuro-navigated TMS was delivered using a Rapid2 stimulator (Magstim) connected to a $70 \mathrm{~mm}$ butterfly coil. Targeted sites in different blocks were over the left and right IPS and over the left and right FEF. To control for any possible nonspecific effects due to lateralized TMS, we identified two suitable sham conditions in a region on the scalp located between IPS and FEF of the left and right hemisphere, respectively. This yielded six different TMS conditions corresponding to the six scalp sites of stimulation (four with active stimulation and two with sham stimulation). The four active TMS sites were localized by means of stereotaxic navigation on individual estimated MRIs obtained through a $3 \mathrm{D}$ warping procedure fitting a high-resolution MRI template with the participant's scalp model and craniometric points (Softaxic, EMS). Neuro-navigation used anatomical Talairach coordinates (Talairach and Tournoux, 1988) obtained by converting the MNI coordinates of the sites of interest from a recent meta-analysis of fMRI studies of eye movements (Zhou and Shu, 2017). Coordinates were $x=-36, y=$ $-1, z=48$ and $x=36 y=-1, z=48$ for left and right FEF, respectively, and $x=-30, y=-53, z=49$ and $x=30, y=-53, z=49$ for left and right IPS, respectively (Fig. $1 C$ ).

Since the existing literature shows that FEF can be localized based on distance from primary motor cortex (M1) (Ro et al., 1999), as an additional step, we measured the anatomical distance on the scalp between the targeted, putative FEF site and the corresponding M1 within each hemisphere. After localizing the area of primary motor cortex that produced the most robust contraction of the contralateral hand, a scalp marking was made on each subject over this location (both over the right and left M1). Then, after localizing the putative FEF hotspots by means of the neuro-navigation approach, as previously described, we calculated the distance between putative FEF and M1 in each hemisphere, separately for each participant. Across subjects, the mean distances between FEF and M1 were $2.76 \mathrm{~cm}$ (right) and $2.78 \mathrm{~cm}$ (left). More importantly, once we obtained our behavioral indexes of TMS-induced modulation of performance (see below), we asked whether such modulation was affected by the distance across individual participants between the two critical sites (M1 and putative FEF). To anticipate, by applying this method and a correlational approach, we obtained evidence to indicate that the effects of TMS across participants tended to be greatest when applied at a certain distance anteriorly from the functionally identified M1 site (see below).

The resting motor threshold (rMT) was determined using a softwarebased "adaptive method" developed by Awiszus (2003) (Motor Threshold Assessment Tool, version 2.0: http://www.clinicalresearcher.org/ software.htm). Any visible muscle twitch was entered in the software as a "valid response." During the experiment, TMS was delivered at $100 \%$ of the individual rMT (mean intensity $=51 \%$ of the maximum stimulator output). For the left FEF/IPS and right FEF/IPS, the coil was initially 
A

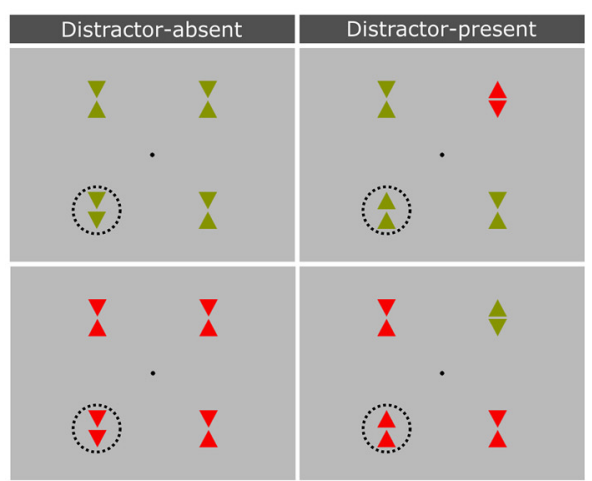

C

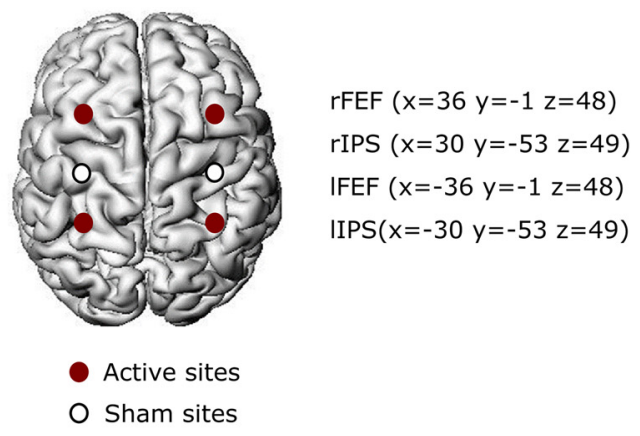

B

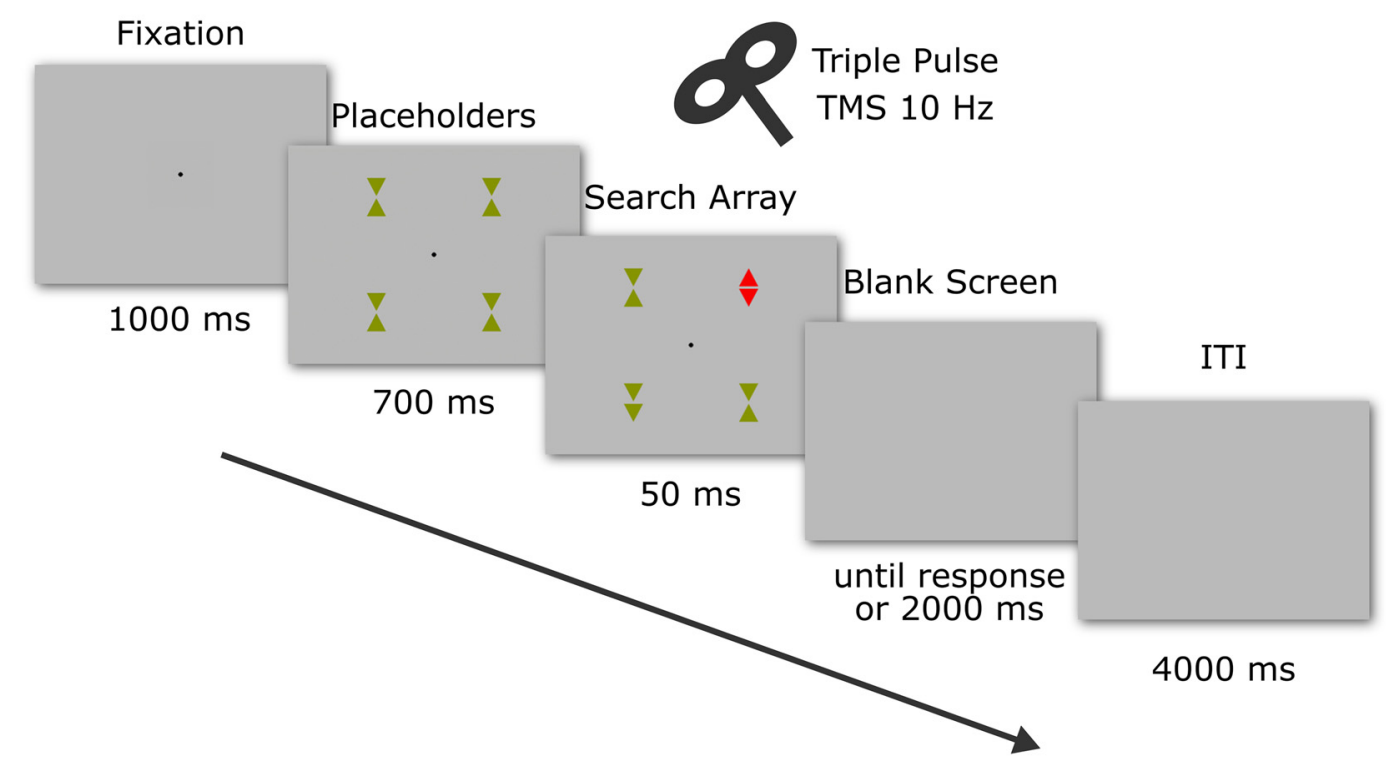

Figure 1. Experimental procedure. $\boldsymbol{A}$, Representation of the four possible types of search arrays used in the task. In $50 \%$ of trials, all display items were of the same color (e.g., red; distractorabsent condition); whereas in the remaining $50 \%$ of trials, three items were of the same color (e.g., red) and the fourth item (additional singleton) was of the alternative color (e.g., green; distractor-present condition). The target, indicated with a dashed circle for graphical purposes only, was defined as the unique double-arrowhead item. $\boldsymbol{B}$, Timeline of an experimental trial. Three TMS pulses were delivered at $10 \mathrm{~Hz}$ (pulse gap of $100 \mathrm{~ms}$ ) starting $100 \mathrm{~ms}$ after search array onset. C, Schematic representation of the stimulated sites: Talairach coordinates were $x=-36, y=-1$, $z=48$ and $x=36, y=-1, z=48$ for left and right FEF, respectively, and $x=-30, y=-53, z=49$ and $x=30, y=-53, z=49$ for left and right IPS, respectively (red circles). The two sham conditions (white circles) were in a region on the scalp located halfway between IPS and FEF of the left and right hemisphere, respectively.

oriented with an angle of $\sim 45^{\circ}$ from the nasion-inion line and the handle pointing outwards, and hence adjusted for each participant to minimize discomfort. For the two sham conditions, the coil was held perpendicular to the scalp to ensure that the magnetic field did not stimulate the underlying cortex. Three TMS pulses were delivered at $10 \mathrm{~Hz}$ (pulse gap of $100 \mathrm{~ms}$ ) starting $100 \mathrm{~ms}$ after search array onset (Fig. 1B). We used triple-pulse $10 \mathrm{~Hz}$ TMS following previous studies showing that these stimulation parameters were effective in modulating the underlying cortical activity (Cohen Kadosh et al., 2010; Saad and Silvanto, 2013). Triple-pulse $10 \mathrm{~Hz}$ TMS starting at $100 \mathrm{~ms}$ covered a time window (100$300 \mathrm{~ms}$ ) that is crucial for attentional capture effects and distractor suppression mechanisms, as shown by human scalp electrophysiology (Jannati et al., 2013; McDonald et al., 2013; Liesefeld et al., 2017). The software Open-Sesame (Mathôt et al., 2012) was used for stimulus presentation, data collection, and TMS triggering.

Statistical analysis. RTs were log-transformed before the analysis to assuage deviations from normality (before transformation: skewness $=$ 2.23 , kurtosis $=5.52$; after transformation: skewness $=-0.05$, kurtosis $=0.44)$. Only correct-response trials were included in the RT analysis (a total of 5.8\% of trials were excluded). Linear mixed-effect models were used as the main statistical procedure (Baayen et al., 2008). We set up each model following the Barr et al. (2013) recommendation to model the maximal random-effects structure justified by the experimental design. Statistical significance was tested with the $F$ test with Satterthwaite approximation of degrees of freedom. All the models were estimated using R (R Development Core Team, 2016) and the lme4 package (version 1.1-12) (Bates et al., 2014). When appropriate, post hoc tests were conducted using the R-package phia (version 0.2-0) (De RosarioMartinez, 2015) and applying the Bonferroni-Holm correction for multiple comparisons.

TMS effects are best understood when comparing each active TMS condition with the corresponding (right or left) sham stimulation. An advantage of having two sham conditions, as opposed to only one, is to subtract out any potential nonspecific TMS effect, such as the lateralized click of the coil associated with the TMS stimulation. Indeed, previous studies demonstrated that the clicking sound of the TMS pulse induces a shift of covert spatial attention to the corresponding side of space, thus facilitating target detection ipsilateral to the stimulation (Duecker and Sack, 2013). In addition to the RT analysis, we conducted an accuracy analysis on all conditions (Table 1). However, since no significant effect of TMS emerged from the accuracy analysis, we will not report those results, except for the sham conditions (see below). Finally, for signifi- 
Table 1. Mean accuracy in percentage for each TMS condition as a function of distractor presence (present vs absent) and brain hemisphere (left vs right) ${ }^{a}$

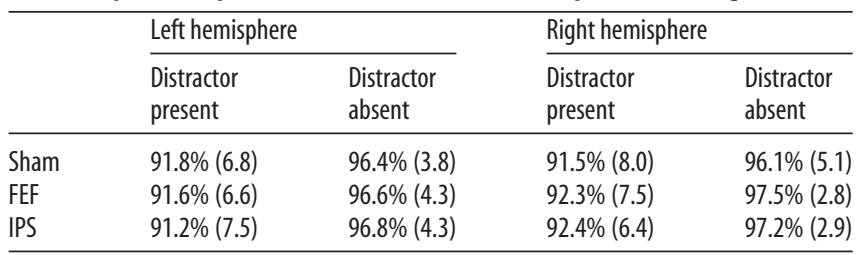

${ }^{a}$ Data are mean (SD).

cant effects, effect size was computed as Cohen's $f^{2}$ (Selya et al., 2012), which uses residual variance from the model to estimate effect size. Since mixed-effect models have two different types of $R^{2}$ (variance explained), the marginal $R^{2}$, which represents the variance explained by the fixed effects, and the conditional $R^{2}$, which represents the variance explained by the entire model, whenever both fixed and random effects were included in the model, two different $f^{2}$ values were calculated for each effect, namely, the $f_{\mathrm{m}}^{2}$ (marginal) and the $f_{\mathrm{c}}^{2}$ (conditional). Nonetheless, for multilevel models, effect sizes calculated using residual variance and proportion of explained variance should be interpreted with caution because the addition of variables to the model may increase residual variance, resulting in negative estimates of explained variance and even of effect size (Snijders and Bosker, 1994).

\section{Results}

\section{Behavioral effects}

To ensure that the adopted paradigm was suitable for the given purposes, we first tested the interfering effect of distracting visual stimuli on task performance in the absence of active TMS stimulation (i.e., restricting the analysis to the sham condition). The brain hemisphere (left vs right), distractor presence (present vs absent), and their interaction were entered as fixed-effect factors in a linear mixed model that predicted log-transformed RTs on correct-response trials. Intercepts and slope for the interaction between brain hemisphere and distractor presence were also included in the model as random-effect factors across participants. This analysis revealed a significant main effect of distractor presence $\left(F_{(1,28.85)}=161.32, p<0.001 ; f_{\mathrm{m}}^{2}=0.071, f_{\mathrm{c}}^{2}=0.14\right)$, reflecting faster responses in the distractor-absent condition (mean $361 \mathrm{~ms}$ ) compared with the distractor-present condition (mean $456 \mathrm{~ms}$ ). The main effect of brain hemisphere was not significant $\left(F_{(1,28.51)}<1, p=0.88\right)$. Importantly, the interaction between distractor presence and brain hemisphere was also nonsignificant $\left(F_{(1,85.98)}<1, p=0.40\right)$, indicating that the effect of distractor did not differ significantly between the two control sham conditions (Fig. 2A). Similarly, a mixed logistic model was estimated using accuracy as the dependent variable. This analysis (Fig. $2 B$ ) revealed a significant main effect of distractor presence $\left(\chi_{(1)}^{2}=\right.$ 34.37, $p<0.001$ ) and no other significant effects (main effect of brain hemisphere: $\chi_{(1)}^{2}<1, p=0.54$; interaction distractor presence by brain hemisphere: $\left.\chi_{(1)}^{2}<1, p=0.94\right)$. Contrast analysis showed that, when a distractor was present, the participants' accuracy rates were lower (94\%) compared with the no-distractor condition (98\%, $z=-4.75, p<0.001$ ) (Fig. $2 B$ ).

As a second step, we evaluated whether, and to what degree, experiencing a distractor in the previous trial modulated distractor interference on the current trial. Indeed, behavioral research has demonstrated larger distractor costs on a given trial $N$ when no distractor was presented (vs when it was presented) on the previous trial N-1 (Geyer et al., 2008; Müller et al., 2010). For this analysis, trials following incorrect-response trials were omitted, as well as incorrect-response trials ( $8.15 \%$ of total trials). Logtransformed RTs were analyzed with a linear mixed model that included brain hemisphere (left vs right), distractor presence (present vs absent), and type of previous trial (distractor-present vs distractor-absent) as fixed-effect factors, and random intercepts and slope for factors brain hemisphere, distractor presence, and type of previous trial within participants. The analysis revealed a significant main effect of distractor presence $\left(F_{(1,28.7)}=\right.$ 152.07, $\left.p<0.001 ; f_{\mathrm{m}}^{2}=0.073, f_{\mathrm{c}}^{2}=0.14\right)$ and a quasi-significant effect of type of previous trial $\left(F_{(1,73.8)}=3.81, p=0.055\right)$. Importantly, and as predicted, the analysis also showed a significant interaction between distractor presence and type of previous trial $\left(F_{(1,7390.2)}=9.37, p=0.002 ; f_{\mathrm{m}}^{2}=0.0005, f_{\mathrm{c}}^{2}=0.001\right)$. This interaction emerged because the distractor cost was larger when a distractor was absent in the previous trial (mean $103 \mathrm{~ms}$ ) compared with when it was present (mean $87 \mathrm{~ms}$ ) (Fig. 2C). Importantly, this effect was not significantly modulated by brain hemisphere, as indicated by a nonsignificant three-way interaction between distractor presence, type of previous trial, and brain hemisphere $\left(F_{(1,7384.9)}<1, p=0.50\right)$. All of the other effects or interactions were not significant: the main effect of brain hemisphere $\left(F_{(1,28)}<1, p=0.82\right)$; the interaction brain hemisphere by distractor presence $\left(F_{(1,7377.3)}<1, p=0.66\right)$; and the interaction brain hemisphere by type of previous trial $\left(F_{(1,7383.4)}<1, p=\right.$ $0.69)$. Finally, a mixed-effect logistic model was estimated using accuracy as the dependent variable. This analysis revealed a significant main effect of distractor $\left(\chi_{(1)}^{2}=35.29, p<0.001\right)$ as before. The interaction between distractor presence and type of previous trial was nonsignificant $\left(\chi_{(1)}^{2}=1.52, p=0.21\right)$. Contrast analysis showed that the cost of distractor did not differ reliably depending on the absence versus presence of a distractor in the previous trial (cost of distraction $=4.9 \%$ and $2.8 \%$, respectively; $z=1.62, p=0.10$; Fig. $2 D)$. No other main effects or interactions were significant: the main effect of brain hemisphere $\left(\chi_{(1)}^{2}<1\right.$, $p=0.70)$; the interaction distractor presence by brain hemisphere $\left(\chi_{(1)}^{2}<1, p=0.78\right)$; the interaction brain hemisphere by type of previous trial $\left(\chi_{(1)}^{2}<1, p=0.64\right)$; and the three-way interaction distractor presence by type of previous trial by brain hemisphere $\left(\chi_{(1)}^{2}=1.12, p=0.29\right)$.

\section{Effect of TMS on visual search}

We tested the effect of TMS on distractor filtering mechanisms using a linear mixed model that predicted log-transformed RTs on correct-response trials. The experimental factors TMS (sham vs FEF vs IPS), distractor presence (present vs absent), brain hemisphere (left vs right), and all their interactions were included as fixed effects. Random coefficients across participants were estimated for intercept and for factors TMS, distractor presence, and brain hemisphere. The omnibus analysis revealed a significant main effect of distractor presence $\left(F_{(1,28.9)}=188.65, p<\right.$ $\left.0.001 ; f_{\mathrm{m}}^{2}=0.065, f_{\mathrm{c}}^{2}=0.13\right)$, indicating that participants were overall faster in the distractor-absent condition (mean $361 \mathrm{~ms}$ ) compared with the distractor-present condition (mean $451 \mathrm{~ms}$ ), with an average distractor cost of $90 \mathrm{~ms}$. Crucially, the three-way interaction TMS by brain hemisphere by distractor presence was significant $\left(F_{(2,24116.7)}=4.20, p=0.01 ; f_{\mathrm{m}}^{2}=0.0002, f_{\mathrm{c}}^{2}=0.0004\right)$ (Table 2). Contrast analysis showed that the effect of the right FEF stimulation (i.e., difference in distractor cost between right sham and right FEF TMS conditions) was significantly different from the effect of the left FEF stimulation (i.e., difference in distractor cost between left sham vs left FEF TMS conditions; $t=2.883, p=$ $0.003)$. This reflected reduced distractor cost following right, but not left, FEF stimulation. The distractor cost associated with IPS stimulation did not significantly differ between brain hemispheres $(t=1.168, p=0.24)$ as well as the difference between IPS 
A

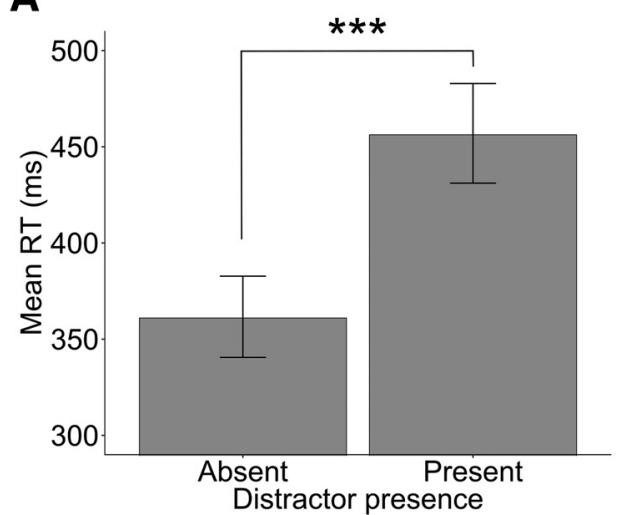

C

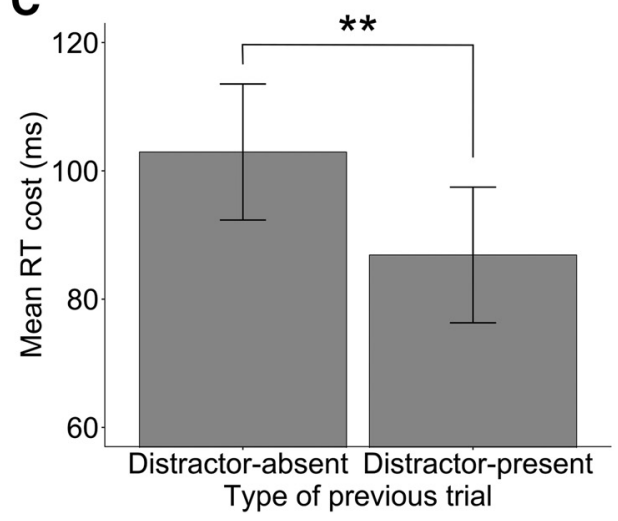

B

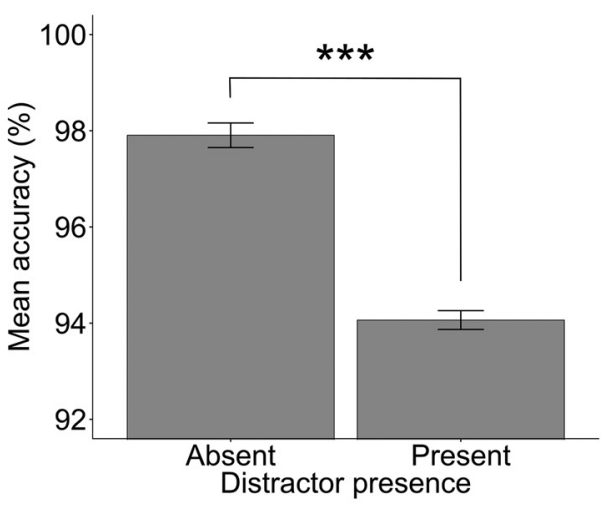

D

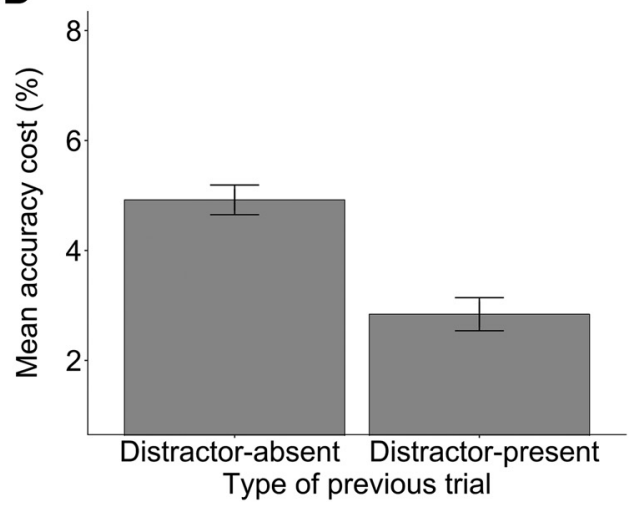

Figure 2. Behavioral effects calculated in the sham condition. $\boldsymbol{A}, \boldsymbol{B}$, Mean RTs $(\boldsymbol{A})$ and mean accuracy $(\boldsymbol{B})$ for trials with a singleton distractor relative to trials without. $\boldsymbol{C}, \boldsymbol{D}$, Difference RTs $(\boldsymbol{C})$ and difference accuracy $(\boldsymbol{D})$ for trials in which the singleton distractor was present relative to absent (distractor cost), shown as a function of the type of previous trial (distractor-present vs distractorabsent). Error bars indicate SEM. ${ }^{* *} p<0.001,{ }^{* *} p<0.01$.

Table 2. Summary of the linear mixed model predicting the effect of TMS on distractor filtering mechanisms as a function of stimulation site and brain hemisphere

\begin{tabular}{llccc}
\hline & Numerator & Denominator & & \\
& DF & DF & \multicolumn{1}{l}{ F } & \multicolumn{1}{c}{$p$} \\
\hline Distractor presence & 1 & 28.9 & 188.6512 & $<0.001^{* * * *}$ \\
TMS & 2 & 29.1 & $<1$ & 0.62 \\
Brain hemisphere & 1 & 28.9 & 1.5934 & 0.22 \\
Distractor presence $\times$ TMS & 2 & 24122.5 & 2.8440 & 0.06 \\
Distractor presence $\times$ brain & 1 & 24123.0 & 2.8074 & 0.09 \\
$\quad$ hemisphere & & & & \\
TMS $\times$ brain hemisphere & 2 & 24117.3 & 8.0674 & $<0.001^{* * *}$ \\
Distractor presence $\times$ TMS & 2 & 24116.7 & 4.2069 & $0.01^{*}$ \\
$\quad \times$ brain hemisphere & & & & \\
\hline${ }^{*} p<0.05 ;{ }^{* * *} p<0.001$. & & & &
\end{tabular}

and FEF TMS stimulation between the left and the right hemisphere $(t=1.718, p=0.09)$ (Fig. $3 A)$.

To further explore the significant three-way interaction, we performed follow-up analyses separately for each hemisphere. The left hemisphere analysis revealed a nonsignificant interaction TMS by distractor presence $\left(F_{(2,53.75)}<1, p=0.85\right)$. On the contrary, the right hemisphere analysis indicated that the interaction TMS by distractor presence was significant $\left(F_{(2,34.89)}=\right.$ $4.58, p=0.017 ; f_{\mathrm{m}}^{2}=0.0005, f_{\mathrm{c}}^{2}=0.0009$ ) (Table 3). Contrast analysis confirmed that the interaction effect reflected a significant decrease in the distractor cost with FEF versus Sham stimulation (the average distractor cost decreased by $23 \mathrm{~ms} ; t=3.023$, $p=0.004)$. The distractor cost did not significantly differ either for the sham versus IPS contrast $(t=1.369, p=0.18)$ or for the IPS versus FEF contrast $(t=1.491, p=0.14)$ (Fig. $3 A$ ).

To further investigate whether the reduction in the distractor cost following right FEF stimulation was due to a relative RT increase in the distractor-absent condition, to a relative RT decrease in the distractor-present condition, or to a combination of both, we conducted post hoc comparisons. These revealed that FEF stimulation reliably reduced RTs compared with sham selectively in the distractor-present condition $\left(\chi_{(1)}^{2}=8.01, p=0.027\right)$. The contrast between FEF and IPS stimulation in the distractorpresent condition revealed a slight trend toward significance $\left(\chi_{(1)}^{2}\right.$ $=5.79, p=0.080)$. All other comparisons were far from significant (all $p$ values $>0.56$; Fig. $3 B, C$ )

An important question to ask is whether any effects of TMS on the distractor cost depend critically on the spatial position of the distractor and/or of the target in the given search display. Therefore, we analyzed RTs in the distractor-present condition as a function of whether the target and distractor appeared in the right or left visual field, that is, ipsilateral or contralateral to the stimulation (only right TMS conditions were considered in this analysis). We implemented a linear mixed model with the experimental factors TMS (sham vs FEF vs IPS), target side (right vs left), and distractor side (right vs left) as fixed effects. The random effect structure included the random intercept for subject, as well as the by-subject random slope for TMS, target side, and distractor side. The analysis indicated a significant interaction between target side and distractor side $\left(F_{(1,5821)}=32.39, p<0.001\right)$, reflecting longer RTs when target and distractor were on the same side (mean $462 \mathrm{~ms}$ ) compared with opposite sides (mean 440 
A

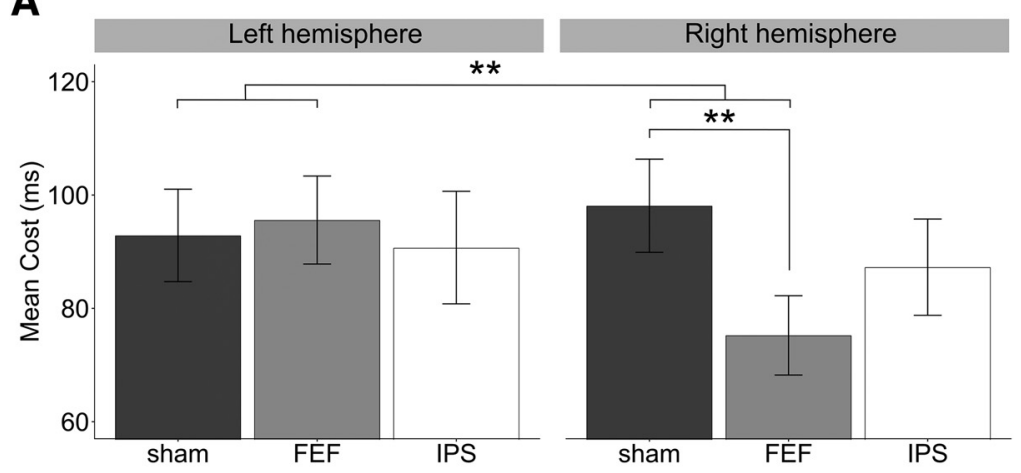

B
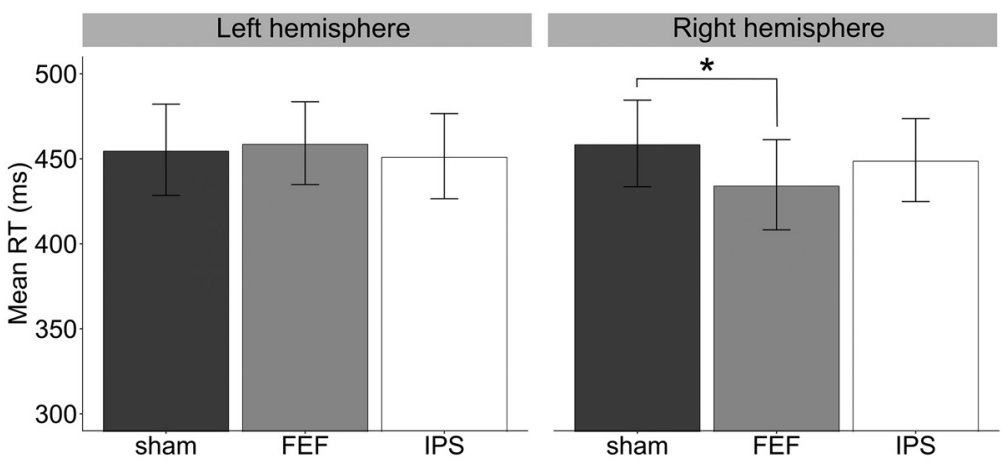

C

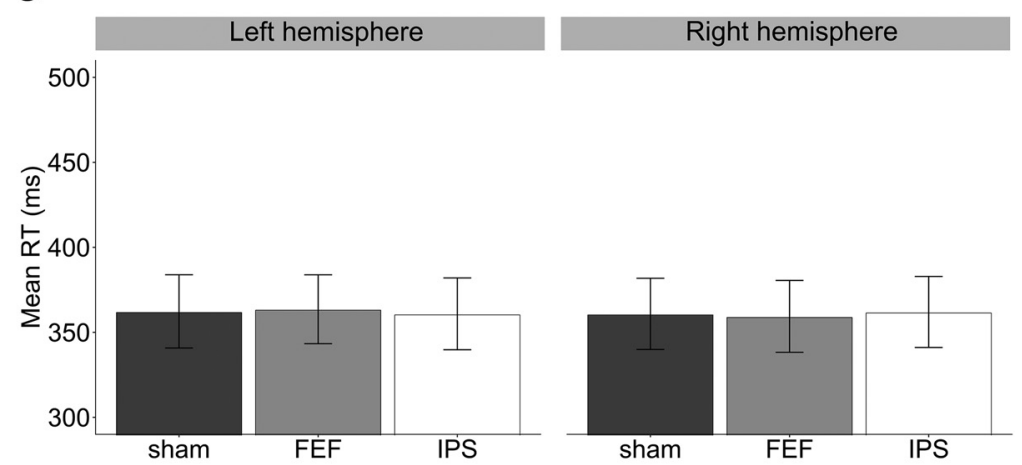

D

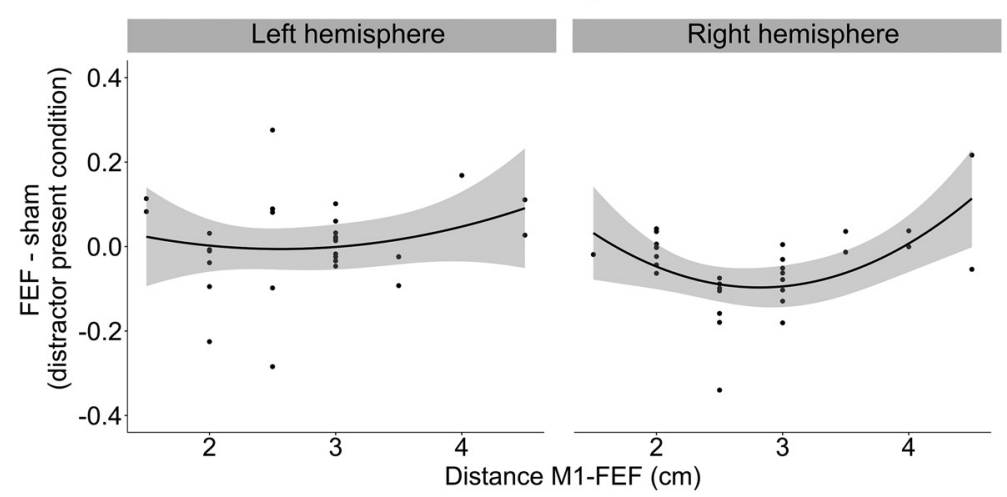

Figure 3. TMS effects on visual search. $\boldsymbol{A}$, Difference RTs for trials in which there was a singleton distractor relative to trials in which it was absent (distractor cost), shown as a function of the TMS site (sham, FEF, and IPS) and brain hemisphere (left, right). The effect of right FEF stimulation was significantly different relative to left FEF stimulation. The distractor cost was significantly reduced after right FEF stimulation compared with its correspondent sham control condition. Error bars indicate SEM. ${ }^{* *} p<0.01$. $\mathrm{ms}$ ), presumably due to greater competition in the former condition relative to the latter. Crucially, TMS did not interact with either target side $\left(F_{(2,5819.9)}<1, p=\right.$ $0.99)$ or distractor side $\left(F_{(2,5823.1)}<1, p=\right.$ $0.48)$, or their interaction $\left(F_{(2,5821.5)}<1\right.$, $p=0.39$ ), indicating that the reduction in the distractor cost associated with right FEF stimulation occurred regardless of the visual field wherein target and distractor stimuli were respectively presented.

An additional analysis was performed to assess whether the chosen coordinates for the FEF stimulation site were optimal with respect to the putative cortical location of FEF at the level of individual participants. Here, coordinates for the FEF site were derived from the existing literature (Zhou and Shu, 2017). However, it is also known that the distance between FEF and M1 is $\sim 2 \mathrm{~cm}$ in humans (Müri et al., 1991; Ro et al., 1999). Because the behavioral effects of TMS over right (and left) FEF varied considerably across participants, and so did the scalp distance between M1 and the stimulated FEF site, we hypothesized that the strongest behavioral effects of FEF stimulation might have occurred in those participants for whom the M1-FEF distance was around the expected, anatomically plausible value of 2 $\mathrm{cm}$ (due to more precise FEF targeting in those participants). To explore this possibility, we fitted a second-order polynomial function predicting the magnitude of the effect reported in the previous analysis ( $\Delta$; i.e., the RT difference between FEF and sham stimulation in the distractorpresent conditions) from the distance between FEF and M1 (d) and on its square (i.e., $\Delta=\beta_{0}+\beta_{1} \mathrm{~d}+\beta_{2} \mathrm{~d}^{2}+\varepsilon$ ). The squared term was included because we expected the distribution of the actual individual FEF-M1 distances to include both smaller and larger values with respect to the putative optimal distance (Müri et al., 1991; Ro et al., 1999), such that the ex-

\footnotetext{
B. Mean RTs for the distractor-present condition, as a function of TMS site and brain hemisphere. Error bars indicate SEM. ${ }^{*} p<0.05$ (Bonferroni-Holm-corrected). TMS over right FEF significantly reduced RTs compared with the corresponding right sham control condition. C, Mean RTs for the distractorabsent condition, as a function of TMS site and brain hemisphere. Error bars indicate SEM. D, Quadratic regression predicting the magnitude of the significant effect (i.e., RT difference between FEF and sham in the distractor-present condition) after the distance between putative FEF and $\mathrm{M} 1$ in individual participants, separately for each hemisphere. The effect of TMS was the strongest when the distance between right putative FEF and right M1 was $\sim 2.77 \mathrm{~cm}$. Shaded area represents the $95 \% \mathrm{Cl}$ of the best-fit line.
}

$\leftarrow$ 
Table 3. Summary of the linear mixed model predicting the effect of TMS on distractor filtering mechanisms, separately computed for left and right hemisphere

\begin{tabular}{llllll}
\hline & \multicolumn{3}{l}{ Numerator } & Denominator & \\
& & DF & DF & $F$ & $p$ \\
\hline Right hemisphere & Distractor presence & 1 & 28.803 & 218.0195 & $<0.001^{* * *}$ \\
& 2 & 28.901 & 2.4004 & 0.10 \\
& TMS & 24.893 & 4.5807 & $0.017^{*}$ \\
& Distractor presence & 2 & & & \\
& $\quad \times$ TMS & & & 150.3842 & $<0.001^{* * *}$ \\
Left hemisphere & Distractor presence & 1 & 28.924 & $<1$ & 0.84 \\
& TMS & 2 & 29.058 & $<1$ & 0.85 \\
& Distractor presence & 2 & 53.750 & $<1$ & \\
\hline & $\times$ TMS & & & & \\
\hline
\end{tabular}

${ }^{*} p<0.05 ;{ }^{* * *} p<0.001$.

pected relationship between those values and the strength of the measured behavioral effects might not be accommodated for by a linear trend only. Interestingly, not only the analysis revealed a significant linear relationship $\left(\beta_{1}=-0.42 ; p=0.01\right)$, indicating that the effect linearly decreased with the distance, but also a significant quadratic effect $\left(\beta_{2}=0.74 ; p=0.006\right)$. These results imply that the effect of TMS was the strongest when the distance between the putative right FEF stimulation site and the right M1 site was $\sim 2.77 \mathrm{~cm}$ and decreased for both shorter and longer distances. This observation indicates that, when the putative FEF site, localized by means of neuro-navigation, was located anteriorly within a certain anatomical distance from M1 (i.e., 2-3 $\mathrm{cm}$ ), the behavioral effects of right FEF stimulation on distractor suppression were strongest, possibly indicating a more precise targeting of the actual FEF location. This finding suggests that the localization of FEF relative to its distance from M1 $(2-3 \mathrm{~cm}$ anterior) might provide a better strategy to target this brain area in future studies. As a control, we performed the same analysis on the left hemisphere. Therefore, we fitted an analogous secondorder polynomial function predicting the magnitude of the effect (i.e., the difference in RT between FEF and sham in the distractorpresent conditions) from the distance between left FEF and M1. This analysis did not reveal any significant effect $\left(\beta_{1}=-0.13\right.$; $p=0.42 ; \beta_{2}=0.26 ; p=0.34$ ) (Fig. $3 D$ ).

Finally, given the significant impact of right FEF stimulation on distractor suppression, we were also interested in testing whether these effects interacted with the recent trial history. To this aim, log-transformed RTs were analyzed with a mixed linear model that included TMS (sham vs FEF), distractor presence (present vs absent), and type of previous trial (distractor-present vs distractor-absent) as fixed-effect factors and intercept, TMS, distractor presence, and type of previous trial as random-effect coefficients across participants. As noted above, for this specific analysis, trials following incorrect-response trials were removed in addition to incorrect-response trials. This analysis revealed a significant main effect of distractor presence $\left(F_{(1,28.5)}=189.92\right.$, $\left.p<0.001 ; f_{\mathrm{m}}^{2}=0.060, f_{\mathrm{c}}^{2}=0.12\right)$. As expected, the analysis confirmed that TMS modulated distractor filtering by unveiling a significant interaction between TMS and distractor presence $\left(F_{(1,7394.0)}=13.07, p<0.001 ; f_{\mathrm{m}}^{2}=0.0008, f_{\mathrm{c}}^{2}=0.001\right)$. The interaction between distractor presence by type of previous trial revealed a trend toward significance $\left(F_{(1,7398.9)}=3.04, p=\right.$ $0.081)$. Interestingly, the three-way interaction TMS by distractor presence by type of previous trial was nearly significant $\left(F_{(1,7403.6)}\right.$ $\left.=3.55, p=0.059 ; f_{\mathrm{m}}^{2}=0.001, f_{\mathrm{c}}^{2}=0.001\right)$. To further explore this quasi-significant interaction, we conducted separate analyses based on the type of previous trial (distractor-absent or distractor-present). When a distractor was present in the previ-

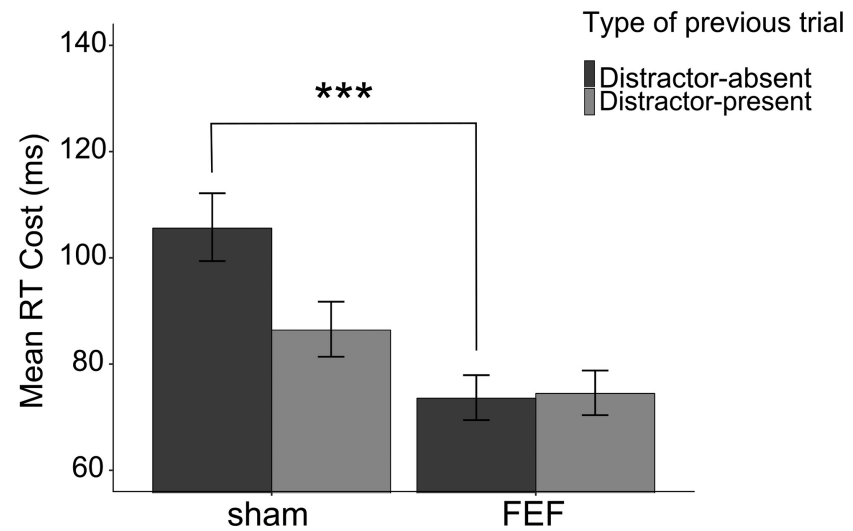

Figure 4. Difference RTs for trials in which the singleton distractor was present minus absent, shown as a function of the type of previous trial (distractor-absent vs distractor-present). Data are shown for sham versus FEF stimulation on the right hemisphere. The relative RT disadvantage following distractor-absent versus distractor-present trials decreased following FEF stimulation compared with sham. Error bars indicate SEM. ${ }^{* * *} p<0.001$.

ous trial, no significant effect of FEF TMS on the distractor cost was found, TMS by distractor presence $\left(F_{(1,3602.3)}=1.71, p=\right.$ $0.19)$. Conversely, when a distractor was absent in the previous trial, TMS significantly modulated the cost of distraction $\left(F_{(1,3743.2)}=15.05, p<0.001 ; f_{\mathrm{m}}^{2}=0.001, f_{\mathrm{c}}^{2}=0.003\right)($ Fig. 4$)$. All of the other effects or interactions did not reach significance (all $p$ values $>0.081$ ). As a control, a similar analysis was also performed comparing sham versus right IPS stimulation. This analysis indicated a main effect of distractor presence $\left(F_{(1,28.6)}=\right.$ $\left.196.58, p<0.001 ; f_{\mathrm{m}}^{2}=0.069, f_{\mathrm{c}}^{2}=0.13\right)$ and a significant interaction distractor presence by type of previous trial $\left(F_{(1,7433,2)}=\right.$ 7.19, $\left.p=0.007 ; f_{\mathrm{m}}^{2}=0.0004, f_{\mathrm{c}}^{2}=0.0008\right)$.

For the right IPS stimulation condition, a nonsignificant effect was found for the interaction TMS by type of previous trial $\left(F_{(1,7432.4)}<1, p=0.39\right)$, albeit a marginally significant effect was obtained for the interaction TMS by distractor presence $\left(F_{(1,7424.1)}=3.10, p=0.07\right)$. The three-way interaction TMS by distractor presence by type of previous trial was nonsignificant $\left(F_{(1,7435.9)}<1, p=0.32\right)$. All of the other effects or interactions did not reach the significance level (all $p$ values $>0.08$ ).

\section{Discussion}

This study sought to ascertain the causal role of two key regions of the dorsal attention network, FEF and IPS, in modulating attentional capture elicited by salient distractor stimuli. Results show that TMS stimulation of the right FEF significantly reduced the behavioral interference caused by a salient singleton distractor during a visual search task. Crucially, this was reliably different from what observed following left FEF stimulation, which had no measurable effect on attentional capture compared with a suitable sham condition. The magnitude of the reduction in distractor interference associated with right FEF stimulation correlated with the anatomical distance between the putative FEF stimulation site and M1 at the single-subject level and peaked in participants for whom such distance was between 2 and $3 \mathrm{~cm}$, compatible with functional localization evidence (Ro et al., 1999). Interestingly, an additional analysis attributed reduction in distractor costs following right FEF stimulation to performance improvements occurring in the distractor-present condition, which attests to the specificity of our results. Perhaps even more interestingly, further analyses indicated that this lessening of distractor costs interacted with modulations of distractor in- 
terference due to intertrial contingencies. In particular, the reduction of distractor interference observed during right FEF stimulation was maximized when the previous trial was a distractor-absent versus distractor-present trial. Together, these results attest to the pivotal role of the right FEF in both limiting attentional capture by salient distractors and modulating historycontingent distractor interference.

Our main result reveals a causal role of right FEF in online distractor filtering. This result aligns nicely with the general idea that the human PFC is responsible for controlling and filtering task-irrelevant information (Shimamura, 2000; Kane and Engle, 2002; de Fockert et al., 2004; de Fockert and Theeuwes, 2012; Geng, 2014; Marini et al., 2016). A recent brain stimulation study showed that tDCS stimulation over bilateral PFC led to a decrease in attentional capture in the additional singleton task (Cosman et al., 2015). Additional evidence that prefrontal regions directly drive the efficient filtering of irrelevant information comes from ERP studies, which identified a frontal ERP component presumably related to distractor suppression (Vogel et al., 2005; Liesefeld et al., 2014; Vissers et al., 2016) (the frontal bias signal). Several fMRI studies have also provided converging evidence on the role of PFC in filtering out irrelevant stimuli. For example, brain activity levels in the PFC correlated with the magnitude of the interfering effects engendered by salient distractors as measured behaviorally (de Fockert et al., 2004; Leber, 2010; de Fockert and Theeuwes, 2012). More relevant for the interpretation of the current results, a brain-behavior relationship has been established between activity levels in the right PFC (right inferior frontal gyrus [rIFG] and right middle frontal gyrus [rMFG]) and behavioral indexes of distractor suppression (Weissman et al., 2006; Demeter et al., 2011; Marini et al., 2016). The distinctive role of a right-lateralized network in attentional control has also been confirmed by neuropsychological evidence, pointing to the rMFG as a crucial node for regulating both top-down and bottom-up attention (see Japee et al., 2015). In the present study, the delivery of repetitive TMS immediately after target (and distractor) presentation may have modulated ongoing activity by increasing neuronal excitability within a network of rightlateralized prefrontal attentional control regions (including rIFG, rMFG, and right FEF). This may have rendered the network prompter to process upcoming visual stimuli, in turn resulting in an optimal instantiation of distraction-filtering mechanisms in the presence of a salient distractor. The existence of strong functional interactions between the FEF, which is part of the dorsal attention network, and the rMFG and rIFG, which are part of the ventral attention network, is supported by several resting-state connectivity studies (Fox et al., 2006; He et al., 2007; Shulman et al., 2009; Asplund et al., 2010). Crucially, DiQuattro and colleagues (DiQuattro and Geng, 2011; DiQuattro et al., 2014) reported that an excitatory pathway from the temporoparietal junction to IFG to FEF contributed to the optimal suppression of irrelevant yet salient distractors. The interpretation of the current results in terms of a facilitating role of TMS in the instantiation of distractor filtering mechanisms fits perfectly with very recent findings in the macaque, demonstrating a shared neural substrate for target selection and distractor suppression in FEF neuronal populations (Cosman et al., 2018). More specifically, this study demonstrated that signatures of (target selection and) salientdistractor filtering in FEF neurons preceded by $\sim 50 \mathrm{~ms}$ posterior ERP signatures of the same process, likely reflecting proactive suppression of the salient distracting stimulus before it can affect neural selection and eventually capture attention (Sawaki and Luck, 2010; see also Gaspelin et al., 2015; Gaspelin and Luck,
2018). In this framework, it is possible that ERP markers of attentional suppression reported in human participants (Liesefeld et al., 2014, 2017) reflect the successful control against distraction, which is implemented by PFC circuitry (and, in particular, right FEF). This would support the general notion that prefrontal-extrastriate projections are responsible for both enhancing task-relevant information and suppressing irrelevant, and especially distracting, information (Serences et al., 2004; Ruff and Driver, 2006; Gazzaley et al., 2007; Cosman et al., 2018).

Our results are compatible with the idea that TMS facilitated the instantiation of distractor suppression. An alternative interpretation is that the right FEF stimulation may have disrupted the attentional bias toward the salient visual feature (the odd color) because this might also lead to reduced capture by the salient distractor. Indeed, it is well known that both FEF and IPS are involved in attentional capture and salience-dependent computations (Corbetta and Shulman, 2002; Serences et al., 2005). Electrophysiological studies with nonhuman primates have consistently demonstrated that visual saliency maps are represented in multiple frontoparietal regions, including LIP (Gottlieb et al., 1998) and FEF (Schall and Hanes, 1993), as well as in subcortical structures, notably the superior colliculus (see White et al., 2017). In the present experiment, TMS may have interfered with attentional mechanisms that are responsible for biasing attention toward salient items. This, in turn, may have weakened attentional capture and reduced the RT cost engendered by salient distractors. In principle, a similar argument could be made for IPS stimulation, which, however, did not significantly modulate the behavioral cost produced by salient distractors. Possibly, the contribution of FEF to attentional biasing toward salient stimuli may be either stronger or more susceptible to TMS-induced modulations than that of IPS. Incidentally, the early involvement of prefrontal regions in bottom-up attention, as shown by research on nonhuman primates, suggests that the identification of salient visual stimuli in parietal and frontal regions may proceed in parallel rather than serially (Thompson et al., 1996; Thomas and Paré, 2007; Katsuki and Constantinidis, 2012). Although the occurrence of a TMS-induced deficit in saliency computation may seem compatible with the available evidence, for at least two reasons, we consider this possibility less likely than our previous interpretation in terms of distraction-filtering mechanisms. First, if saliency computation were compromised by the right FEF TMS stimulation, then we might expect a performance cost in the distractor-absent condition, too, because saliency computation should have also supported target selection, in addition to attentional capture by the singleton distractor. However, our data indicated no TMS-induced modulation of performance in the distractor-absent condition. Second, an interpretation in terms of saliency computation does not seem to provide an obvious account for the effects of TMS on modulations of performance related to cross-trial contingencies (see below). For these reasons, we favor an interpretation whereby right FEF TMS modulated mechanisms responsible for the effective filtering of salient distractors.

The negative results we found for the IPS stimulation may appear at odds with those reported by Hodsoll et al. (2009), as they described a significant reduction in the distractor cost following right PPC TMS. However, crucial differences in the TMS protocol may account for the discrepant results. Hodsoll et al. (2009) used an offline, $1 \mathrm{~Hz}$ TMS approach, known to give rise to prolonged cortical inhibition at the site of stimulation (Chen et al., 1997; Oliveri et al., 2005). Instead, our time-locked, $10 \mathrm{~Hz}$ stimulation protocol may have been suboptimal for inducing 
clear-cut behavioral changes following IPS stimulation. In line with this interpretation, previous ERP studies demonstrated a sequential involvement of FEF and IPS during distractor-filtering tasks, with frontal signals preceding those detected over parietal areas (Grent-'t-Jong and Woldorff, 2007; Leblanc et al., 2008; Brignani et al., 2009; Ptak et al., 2011; Shomstein et al., 2012; Liesefeld et al., 2014). Therefore, we cannot exclude the possibility that the early stimulation (between 100 and 300 ms after display onset) applied in the current study was adequate for affecting FEF but not IPS activity, and that a later or more prolonged stimulation may reveal the involvement of IPS in the current behavioral context. These remain open possibilities for future studies to explore.

The present results are also compatible with the proposed general role of the frontoparietal attention network in modulating the responsiveness of visual cortical regions (Grosbras and Paus, 2003; Moore and Armstrong, 2003; Silvanto et al., 2006, 2009; Bisley and Goldberg, 2010; Noudoost et al., 2010; Baluch and Itti, 2011; Buschman and Kastner, 2015; Scolari et al., 2015; Marini et al., 2016). The available evidence suggests that suppression mechanisms may be implemented through prefrontaldriven modulations of sensory processing (Serences et al., 2004; Gazzaley et al., 2007; Seidl et al., 2012), similarly to sensory enhancements of target features (Reynolds and Chelazzi, 2004; Chelazzi et al., 2011). Moreover, recent findings both in humans (Michalareas et al., 2016; Wang et al., 2016; Popov et al., 2017) and nonhuman primates (van Kerkoerle et al., 2014; Bastos et al., 2015) suggest that FEF may exert top-down control by modulating visual oscillatory alpha-band activity in sensory areas. Recently, Popov et al. (2017) used MEG to show that right FEF, but not left FEF, exerts top-down control on stimulus processing in visual cortex. This FEF hemispheric asymmetry fits well with our current results and is complemented by the typical finding that TMS modulates visual task performance in both (left and right) hemifields when applied over the right FEF, and in the right hemifield only when applied over the left FEF (Grosbras and Paus, 2003; Smith et al., 2005; Silvanto et al., 2006). In general, the present results concur with the large body of evidence suggesting a right hemispheric dominance for the control of visuo-spatial attention in the FEF (Grosbras and Paus, 2003; Silvanto et al., 2006; Capotosto et al., 2009; Duecker et al., 2013; Marshall et al., 2015; Wang et al., 2016).

Interestingly, the beneficial effects of right FEF stimulation on distractor suppression seem to interact with modulations of distractor costs that are related to the presence versus absence of a distractor in the preceding trial, suggesting a role of the right frontal attention network in the intertrial, history-dependent regulation of distraction-filtering mechanisms. More specifically, in the sham condition, the interfering effect of the distractor was greater on trials following a distractor-absent trial compared with trials following a distractor-present trial, an observation that has been reported previously (Geyer et al., 2008). The TMS stimulation of right FEF eliminated the relative disadvantage of having experienced a distractor-absent condition in the previous trial, which could be alternatively interpreted as if TMS mimicked the advantage of having encountered a distractor-present condition. We have already suggested that the effect of TMS on neuronal excitability might have consisted of an increase of activity in neuronal populations implementing distractor-filtering mechanisms in the current trial. In addition, TMS may have also facilitated the sustained maintenance of distraction-filtering mechanisms through the subsequent trial, hence resulting in a reduction of the distractor cost. This idea can be accommodated in the framework of the interactions between the conflict monitoring system (Botvinick et al., 2001) and the frontoparietal attention network. According to this framework, when response conflict occurs, the ACC signals an increased demand for cognitive control, which leads to an enhancement of top-down control mediated by the dorsal frontoparietal network (FEF and IPS) (see Walsh et al., 2011) on the subsequent trial (Carter et al., 2000; Casey et al., 2000; Botvinick et al., 2001). From an anatomical and physiological perspective, this is supported by the existence of direct axonal projections between ACC and both FEF (Huerta et al., 1987; Stanton et al., 1993) and PPC (Pandya et al., 1981; Hampson et al., 2006). Perhaps similarly to response conflict, also the presence of a salient distractor may trigger the ACC reactively (see Seeley et al., 2007) and then engage greater attentional control proactively in preparation for the subsequent trial, thus leading to reduced distractor costs. Here, magnetic stimulation may have primed the attention control network in a way that mimicked the proactive adjustments occurring spontaneously after distractorpresent trials. This, in turn, would have resulted in a reduced distractor cost in the subsequent trial. The idea that right FEF TMS may have promoted proactive trial-to-trial control finds support in a study by Leber (2010), showing that stronger pretrial activity in prefrontal areas was associated with reduced distractor interference by a salient irrelevant distractor.

As a cautionary note, the design of our experiment is not optimally suited to draw firm conclusions concerning the effect of TMS on intertrial modulations. Indeed, by applying TMS pulses on every trial, we cannot cleanly disentangle whether the reported effects were due to an influence of TMS on the current trial $(\mathrm{N})$ or instead on the previous trial $(\mathrm{N}-1)$. Regardless of this limitation, our results demonstrated that following right FEF stimulation the relative disadvantage of having experienced a distractor-absent condition in the previous trial was completely eliminated. This finding can be interpreted in two alternative ways: On the one hand, TMS on the current trial $\mathrm{N}$ may have strengthened reactive (online) mechanisms to deal with distraction, those very mechanisms that are especially important when the system has not been alerted by a distractor on the preceding trial. Alternatively, TMS on the previous (distractor-absent) trial may have proactively primed the attention control network, mimicking the modulation occurring spontaneously on distractorpresent trials. Future and more sensitive TMS experimental designs will help clarify this distinction.

In conclusion, with a systematic approach, the present findings indicate a causal role of the right FEF in adjusting attentional filtering mechanisms. We propose that the TMS intervention engaged the cortical network that controls and regulates mechanisms for limiting interference from irrelevant, attentioncapturing distractor stimuli. More specifically, TMS may have shielded the network from the impact of potential distractor interference, both by facilitating online distractor filtering mechanisms and by modulating intertrial adjustments. Moreover, our results suggest that not only right FEF is directly involved in the reactive control mechanisms that deal with distracting stimuli after their appearance, but it also mediates attentional control mechanisms that are modulated on a trial-by-trial basis. Future research will have to elucidate the contribution to distraction filtering of other cortical regions, notably ventral attention network regions, such as IFG/MFG in the frontal lobe and temporoparietal junction in the parietal lobe, and will clarify whether the role of ventral regions is similar or distinguishable from the role of the dorsal attention network characterized here. 


\section{References}

Asplund CL, Todd JJ, Snyder AP, Marois R (2010) A central role for the lateral prefrontal cortex in goal-directed and stimulus-driven attention. Nat Neurosci 13:507-512.

Awiszus F (2003) TMS and threshold hunting. Suppl Clin Neurophysiol 56:13-23.

Baayen RH, Davidson DJ, Bates DM (2008) Mixed-effects modeling with crossed random effects for subjects and items. J Mem Lang 59:390-412.

Baluch F, Itti L (2011) Mechanisms of top-down attention. Trends Neurosci 34:210-224.

Barr DJ, Levy R, Scheepers C, Tily HJ (2013) Random effects structure for confirmatory hypothesis testing: keep it maximal. J Mem Lang 68:255-278.

Bastos AM, Vezoli J, Bosman CA, Schoffelen JM, Oostenveld R, Dowdall JR, De Weerd P, Kennedy H,Fries P (2015) Visual areas exert feedforward and feedback influences through distinct frequency channels. Neuron 85:390-401.

Bates D, Maechler M, Bolker B, Walker S (2014) lme4: linear mixed-effect models using Eigen and S4 (R package version 1.1-7). Available at http://cran.r-project.org/package $=$ lme4.

Bisley JW, Goldberg ME (2010) Attention, intention, and priority in the parietal lobe. Annu Rev Neurosci 33:1-21.

Botvinick MM, Braver TS, Barch DM, Carter CS, Cohen JD (2001) Conflict monitoring and cognitive control. Psychol Rev 108:624-652.

Brignani D, Guzzon D, Marzi CA, Miniussi C (2009) Attentional orienting induced by arrows and eye-gaze compared with an endogenous cue. Neuropsychologia 47:370-381.

Buschman TJ, Kastner S (2015) From behavior to neural dynamics: an integrated theory of attention. Neuron 88:127-144.

Capotosto P, Babiloni C, Romani GL, Corbetta M (2009) Frontoparietal cortex controls spatial attention through modulation of anticipatory alpha rhythms. J Neurosci 29:5863-5872.

Carter CS, Macdonald AM, Botvinick M, Ross LL, Stenger VA, Noll D, Cohen JD (2000) Parsing executive processes: strategic vs. evaluative functions of the anterior cingulate cortex. Proc Natl Acad Sci U S A 97:1944-1948.

Casey BJ, Thomas KM, Welsh TF, Badgaiyan RD, Eccard CH, Jennings JR, Crone EA (2000) Dissociation of response conflict, attentional selection, and expectancy with functional magnetic resonance imaging. Proc Natl Acad Sci U S A 97:8728-8733.

Chelazzi L, Della Libera C, Sani I, Santandrea E (2011) Neural basis of visual selective attention. Wiley Interdiscip Rev Cogn Sci 2:392-407.

Chelazzi L, Marini F, Pascucci D, Turatto M (2019) Getting rid of visual distractors: the why, when, how, and where. Curr Opin Psychol 29:135147.

Chen R, Classen J, Gerloff C, Celnik P, Wassermann EM, Hallett M, Cohen LG (1997) Depression of motor cortex excitability by low-frequency transcranial magnetic stimulation. Neurology 48:1398-1403.

Cohen Kadosh RC, Muggleton N, Silvanto J, Walsh V (2010) Double dissociation of format-dependent and number-specific neurons in human parietal cortex. Cereb Cortex 20:2166-2171.

Corbetta M, Shulman GL (2002) Control of goal-directed and stimulusdriven attention in the brain. Nat Rev Neurosci 3:201-215.

Cosman JD, Atreya PV, Woodman GF (2015) Transient reduction of visual distraction following electrical stimulation of the prefrontal cortex. Cognition 145:73-76.

Cosman JD, Lowe KA, Zinke W, Woodman GF, Schall JD (2018) Prefrontal control of visual distraction. Curr Biol 28:414-420.e3.

De Rosario-Martinez (2015) phia: post-hoc interaction analysis. https:// cran.r-project.org/package $=$ phia.

de Fockert JW, Theeuwes J (2012) Role of frontal cortex in attentional capture by singleton distractors. Brain Cogn 80:367-373.

de Fockert J, Rees G, Frith C, Lavie N (2004) Neural correlates of attentional capture in visual search. J Cogn Neurosci 16:751-759.

Demeter E, Hernandez-Garcia L, Sarter M, Lustig C (2011) Challenges to attention: a continuous arterial spin labeling (ASL) study of the effects of distraction on sustained attention. Neuroimage 54:1518-1529.

Di Caro V, Theeuwes J, Della Libera C (2019) Suppression history of spatial locations biases attentional and oculomotor control. J Vis 18:477.

DiQuattro NE, Geng JJ (2011) Contextual knowledge configures attentional control networks. J Neurosci 31:18026-18035.

DiQuattro NE, Sawaki R, Geng JJ (2014) Effective connectivity during feature-based attentional capture: evidence against the attentional reorienting hypothesis of TPJ. Cereb Cortex 24:3131-3141.

Donohue SE, Bartsch MV, Heinze HJ, Schoenfeld MA, Hopf JM (2018) Cortical mechanisms of prioritizing selection for rejection in visual search. J Neurosci 38:4738-4748.

Duecker F, Sack AT (2013) Pre-stimulus sham TMS facilitates target detection. PLoS One 8:e57765.

Duecker F, Formisano E, Sack AT (2013) Hemispheric differences in the voluntary control of spatial attention: direct evidence for a righthemispheric dominance within frontal cortex. J Cogn Neurosci 25:1332-1342.

Egeth HE, Leonard CJ, Leber AB (2010) Why salience is not enough: reflections on top-down selection in vision. Acta Psychol (Amst) 135:130-132.

Ferrante O, Patacca A, Di Caro V, Della Libera C, Santandrea E, Chelazzi L (2018) Altering spatial priority maps via statistical learning of target selection and distractor filtering. Cortex 102:67-95.

Folk CL, Remington R (2010) A critical evaluation of the disengagement hypothesis. Acta Psychol 135:103-105.

Forster S, Lavie N (2008) Failures to ignore entirely irrelevant distractors: the role of load. J Exp Psychol Appl 14:73-83.

Fox MD, Corbetta M, Snyder AZ, Vincent JL, Raichle ME (2006) Spontaneous neuronal activity distinguishes human dorsal and ventral attention systems. Proc Natl Acad Sci U S A 103:10046-10051.

Gaspar JM, McDonald JJ (2014) Suppression of salient objects prevents distraction in visual search. J Neurosci 34:5658-5666.

Gaspelin N, Luck SJ (2018) The role of inhibition in avoiding distraction by salient stimuli. Trends Cogn Sci 22:79-92.

Gaspelin N, Leonard CJ, Luck SJ (2015) Direct evidence for active suppression of salient-but-irrelevant sensory inputs. Psychol Sci 26:1740-1750.

Gazzaley A, Rissman J, Cooney J, Rutman A, Seibert T, Clapp W, D’Esposito M (2007) Functional interactions between prefrontal and visual association cortex contribute to top-down modulation of visual processing. Cereb Cortex 17 [Suppl 1]:i125-i135.

Geng JJ (2014) Attentional mechanisms of distractor suppression. Curr Dir Psychol Sci 23:147-153.

Geyer T, Müller HJ, Krummenacher J (2008) Expectancies modulate attentional capture by salient color singletons. Vision Res 48:1315-1326.

Goschy H, Bakos S, Müller HJ, Zehetleitner M (2014) Probability cueing of distractor locations: both intertrial facilitation and statistical learning mediate interference reduction. Front Psychol 5:1195.

Gottlieb JP, Kusunoki M, Goldberg ME (1998) The representation of visual salience in monkey parietal cortex. Nature 391:481-484.

Grent-'t-Jong T, Woldorff MG (2007) Timing and sequence of brain activity in top-down control of visual-spatial attention. PLoS Biol 5:e12.

Grosbras MH, Paus T (2003) Transcranial magnetic stimulation of the human frontal eye field facilitates visual awareness. Eur J Neurosci 18:31213126.

Hampson M, Driesen NR, Skudlarski P, Gore JC, Constable RT (2006) Brain connectivity related to working memory performance. J Neurosci 26:13338-13343.

He BJ, Snyder AZ, Vincent JL, Epstein A, Shulman GL, Corbetta M (2007) Breakdown of functional connectivity in frontoparietal networks underlies behavioral deficits in spatial neglect. Neuron 53:905-918.

Hodsoll J, Mevorach C, Humphreys GW (2009) Driven to less distraction: rTMS of the right parietal cortex reduces attentional capture in visual search. Cereb Cortex 19:106-114.

Huerta MF, Krubitzer LA, Kaas JH (1987) Frontal eye field as defined by intracortical microstimulation in squirrel monkeys, owl monkeys, and macaque monkeys: II. Cortical connections. J Comp Neurol 265:332-361.

Ipata AE, Gee AL, Gottlieb J, Bisley JW, Goldberg ME (2006) LIP responses to a popout stimulus are reduced if it is overtly ignored. Nat Neurosci 9:1071-1076

Jannati A, Gaspar JM, McDonald JJ (2013) Tracking target and distractor processing in fixed-feature visual search: evidence from human electrophysiology. J Exp Psychol Hum Percept Perform 39:1713-1730.

Japee S, Holiday K, Satyshur MD, Mukai I, Ungerleider LG (2015) A role of right middle frontal gyrus in reorienting of attention: a case study. Front Syst Neurosci 9:1-16.

Jonides J, Yantis S (1988) Uniqueness of abrupt visual onset in capturing attention. Percept Psychophys 43:346-354.

Kajimura S, Nomura M (2015) Decreasing propensity to mind-wander with transcranial direct current stimulation. Neuropsychologia 75:533-537. 
Kane MJ, Engle RW (2002) The role of prefrontal cortex in workingmemory capacity, executive attention, and general fluid intelligence: an individual differences perspective. Psychon Bull Rev 9:637-671.

Katsuki F, Constantinidis C (2012) Early involvement of prefrontal cortex in visual bottom-up attention. Nat Neurosci 15:1160-1166.

Kerns JG, Cohen JD, MacDonald AW 3rd, Cho RY, Stenger VA, Carter CS (2004) Anterior cingulate conflict monitoring and adjustments in control. Science 303:1023-1026.

Leber AB (2010) Neural predictors of within-subject fluctuations in attentional control. J Neurosci 30:11458-11465.

Leblanc E, Prime DJ, Jolicoeur P (2008) Tracking the location of visuospatial attention in a contingent capture paradigm. J Cogn Neurosci 20:657-671.

Lee J, Geng JJ (2017) Idiosyncratic patterns of representational similarity in prefrontal cortex predict attentional performance. J Neurosci 37:12571268.

Liesefeld AM, Liesefeld HR, Zimmer HD (2014) Intercommunication between prefrontal and posterior brain regions for protecting visual working memory from distractor interference. Psychol Sci 25:325-333.

Liesefeld HR, Liesefeld AM, Töllner T, Müller HJ (2017) Attentional capture in visual search: capture and post-capture dynamics revealed by EEG. Neuroimage 156:166-173.

Marini F, Chelazzi L, Maravita A (2013) The costly filtering of potential distraction: evidence for a supramodal mechanism. J Exp Psychol Gen 142:906-922.

Marini F, Demeter E, Roberts KC, Chelazzi L, Woldorff MG (2016) Orchestrating proactive and reactive mechanisms for filtering distracting information: brain-behavior relationships revealed by a mixed-design fMRI study. J Neurosci 36:988-1000.

Marshall TR, O'Shea J, Jensen O, Bergmann TO (2015) Frontal eye fields control attentional modulation of alpha and gamma oscillations in contralateral occipitoparietal cortex. J Neurosci 35:1638-1647.

Mathôt S, Schreij D, Theeuwes J (2012) OpenSesame: an open-source, graphical experiment builder for the social sciences. Behav Res Methods 44:314-324.

McDonald JJ, Green JJ, Jannati A, Di Lollo V (2013) On the electrophysiological evidence for the capture of visual attention. J Exp Psychol Hum Percept Perform 39:849-860.

Melloni L, van Leeuwen S, Alink A, Müller NG (2012) Interaction between bottom-up saliency and top-down control: how saliency maps are created in the human brain. Cereb Cortex 22:2943-2952.

Michalareas G, Vezoli J, van Pelt S, Schoffelen JM, Kennedy H, Fries P (2016) Alpha-beta and gamma rhythms subserve feedback and feedforward influences among human visual cortical areas. Neuron 89:384-397.

Moore T, Armstrong KM (2003) Selective gating of visual signals by microstimulation of frontal cortex. Nature 421:370-373.

Müller HJ, Töllner T, Zehetleitner M, Geyer T, Rangelov D, Krummenacher J (2010) Dimension-based attention modulates feed-forward visual processing. Acta Psychol (Amst) 135:117-122.

Müller NG, Ebeling D (2008) Attention-modulated activity in visual cortex: more than a simple 'spotlight.' Neuroimage 40:818-827.

Müri RM, Hess CW, Meienberg O (1991) Transcranial stimulation of the human frontal eye field by magnetic pulses. Exp Brain Res 86:219-223.

Neo G, Chua FK (2006) Capturing focused attention. Percept Psychophys 68:1286-1296.

Noudoost B, Chang MH, Steinmetz NA, Moore T (2010) Top-down control of visual attention. Curr Opin Neurobiol 20:183-190.

Oliveri M, Koch G, Torriero S, Caltagirone C (2005) Increased facilitation of the primary motor cortex following $1 \mathrm{~Hz}$ repetitive transcranial magnetic stimulation of the contralateral cerebellum in normal humans. Neurosci Lett 376:188-193.

Pandya DN, Van Hoesen GW, Mesulam MM (1981) Efferent connections of the cingulate gyrus in the rhesus monkey. Exp Brain Res 42:319-330.

Pascual-Leone A, Walsh V, Rothwell J (2000) Transcranial magnetic stimulation in cognitive neuroscience-virtual lesion, chronometry, and functional connectivity. Curr Opin Neurobiol 10:232-237.

Pascucci D, Turatto M (2015) The distracting impact of repeated visible and invisible onsets on focused attention. J Exp Psychol Hum Percept Perform 41:879-892.

Popov T, Kastner S, Jensen O (2017) FEF-controlled alpha delay activity precedes stimulus-induced gamma-band activity in visual cortex. J Neurosci 37:4117-4127.
Ptak R, Camen C, Morand S, Schnider A (2011) Early event-related cortical activity originating in the frontal eye fields and inferior parietal lobe predicts the occurrence of correct and error saccades. Hum Brain Mapp 32:358-369.

R Development Core Team (2016) R: a language and environment for statistical computing. R Foundation for Statistical Computing, Vienna.

Reynolds JH, Chelazzi L (2004) Attentional modulation of visual processing. Annu Rev Neurosci 27:611-647.

Rossi S, Hallett M, Rossini PM, Pascual-Leone A (2009) Safety, ethical considerations, and application guidelines for the use of transcranial magnetic stimulation in clinical practice and research. Clin Neurophysiol 120:2008-2039.

Ro T, Cheifet S, Ingle H, Shoup R, Rafal R (1999) Localization of the human frontal eye fields and motor hand area with transcranial magnetic stimulation and magnetic resonance imaging. Neuropsychologia 37:225-231.

Ruff CC, Driver J (2006) Attentional preparation for a lateralized visual distractor: behavioral and fMRI evidence. J Cogn Neurosci 18:522-538.

Saad E, Silvanto J (2013) How visual short-term memory maintenance modulates the encoding of external input: evidence from concurrent visual adaptation and TMS. Neuroimage 72:243-251.

Sauter M, Liesefeld HR, Zehetleitner M, Müller HJ (2018) Region-based shielding of visual search from salient distractors: target detection is impaired with same, but not different-dimension distractors. Atten Percept Psychophys 80:622-642.

Sauter M, Liesefeld HR, Müller HJ (2019) Learning to suppress salient distractors in the target dimension: region-based inhibition is persistent and transfers to distractors in a nontarget dimension. J Exp Psychol Learn Mem Cogn. Advance online publication. Retrieved January 28, 2019. doi: $10.1037 / x l m 0000691$

Sawaki R, Luck SJ (2010) Capture versus suppression of attention by salient singletons: electrophysiological evidence for an automatic attend-to-me signal. Atten Percept Psychophys 72:1455-1470.

Schall JD, Hanes DP (1993) Neural basis of saccade target selection in frontal eye field during visual search. Nature 366:467-469.

Scolari M, Seidl-Rathkopf KN, Kastner S (2015) Functions of the human frontoparietal attention network: evidence from neuroimaging. Curr Opin Behav Sci 1:32-39.

Seeley WW, Menon V, Schatzberg AF, Keller J, Glover GH, Kenna H, Reiss AL,Greicius MD (2007) Dissociable intrinsic connectivity networks for salience processing and executive control. J Neurosci 27:2349-2356.

Seidl KN, Peelen MV, Kastner S (2012) Neural evidence for distracter suppression during visual search in real-world scenes. J Neurosci 32:1181211819.

Selya AS, Rose JS, Dierker LC, Hedeker D, Mermelstein RJ (2012) A practical guide to calculating Cohen's $\mathrm{f} 2$, a measure of local effect size, from PROC MIXED. Front Psychol 3:1-6.

Serences JT, Yantis S, Culberson A, Awh E (2004) Preparatory activity in visual cortex indexes distractor suppression during covert spatial orienting. J Neurophysiol 92:3538-3545.

Serences JT, Shomstein S, Leber AB, Golay X, Egeth HE, Yantis S (2005) Coordination of voluntary and stimulus-driven attentional control in human cortex. Psychol Sci 16:114-122.

Shimamura AP (2000) The role of the prefrontal cortex in dynamic filtering. Psychobiology 28:207-218.

Shomstein S, Kravitz DJ, Behrmann M (2012) Attentional control: temporal relationships within the fronto-parietal network. Neuropsychologia 50:1202-1210.

Shulman GL, Astafiev SV, Franke D, Pope DL, Snyder AZ, McAvoy MP, Corbetta M (2009) Interaction of stimulus-driven reorienting and expectation in ventral and dorsal frontoparietal and basal ganglia-cortical networks. J Neurosci 29:4392-4407.

Silvanto J, Cattaneo Z (2017) Common framework for 'virtual lesion' and state-dependent TMS: the facilitatory/suppressive range model of online TMS effects on behavior. Brain Cogn 119:32-38.

Silvanto J, Pascual-Leone A (2008) State-dependency of transcranial magnetic stimulation. Brain Topogr 21:1-10.

Silvanto J, Lavie N, Walsh V (2006) Stimulation of the human frontal eye fields modulates sensitivity of extrastriate visual cortex. J Neurophysiol 96:941-945.

Silvanto J, Muggleton N, Lavie N, Walsh V (2009) The perceptual and functional consequences of parietal top-down modulation on the visual cortex. Cereb Cortex 19:327-330. 
Smith DT, Jackson SR, Rorden C (2005) Transcranial magnetic stimulation of the left human frontal eye fields eliminates the cost of invalid endogenous cues. Neuropsychologia 43:1288-1296.

Snijders TA, Bosker RJ (1994) Modeled variance in two-level models. Sociol Methods Res 22:342-363.

Soutschek A, Taylor PC, Müller HJ, Schubert T (2013) Dissociable networks control conflict during perception and response selection: a transcranial magnetic stimulation study. J Neurosci 33:5647-5654.

Stanton GB, Bruce CJ, Goldberg ME (1993) Topography of projections to the frontal lobe from the macaque frontal eye fields. J Comp Neurol 330:286-301.

Suzuki M, Gottlieb J (2013) Distinct neural mechanisms of distractor suppression in the frontal and parietal lobe. Nat Neurosci 16:98-104.

Talairach J, Tournoux P (1988) Co-planar stereotaxic atlas of the human brain: 3-dimensional proportional system: an Approach to cerebral imaging. Stuttgart, Germany: Theime.

Talsma D, Coe B, Munoz DP, Theeuwes J (2010) Brain structures involved in visual search in the presence and absence of color singletons. J Cogn Neurosci 22:761-774.

Theeuwes J (1992) Perceptual selectivity for color and form. Percept Psychophys 51:599-606.

Theeuwes J, Burger R (1998) Attentional control during visual search: the effect of irrelevant singletons. J Exp Psychol Hum Percept Perform 24: $1342-1353$

Theeuwes J, Godljn R (2002) Irrelevant singletons capture attention: evidence from inhibition of return. Percept Psychophys 64:764-770.

Thomas NW, Paré M (2007) Temporal processing of saccade targets in parietal cortex area LIP during visual search. J Neurophysiol 97:942-947.

Thompson KG, Hanes DP, Bichot NP, Schall JD (1996) Perceptual and motor processing stages identified in the activity of macaque frontal eye field neurons during visual search. J Neurophysiol 76:4040-4055.

Tommasi G, Fiorio M, Yelnik J, Krack P, Sala F, Schmitt E, Fraix V, Bertolasi L, Le Bas JF, Ricciardi GK, Fiaschi A, Theeuwes J, Pollak P, Chelazzi L (2015) Disentangling the role of cortico-basal ganglia loops in top-down and bottom-up visual attention: an investigation of attention deficits in Parkinson disease. J Cogn Neurosci 27:1215-1237.

Turatto M, Bonetti F, Pascucci D, Chelazzi L (2018a) Desensitizing the attention system to distraction while idling: a new latent learning phenomenon in the visual attention domain. J Exp Psychol Gen 147:1827-1850.

Turatto M, Bonetti F, Pascucci D (2018b) Filtering visual onsets via habitu- ation: a context-specific long-term memory of irrelevant stimuli. Psychon Bull Rev 25:1028-1034.

van Kerkoerle T, Self MW, Dagnino B, Gariel-Mathis MA, Poort J, van der Togt C, Roelfsema PR (2014) Alpha and gamma oscillations characterize feedback and feedforward processing in monkey visual cortex. Proc Natl Acad Sci U S A 111:14332-14341.

Vissers ME, van Driel J, Slagter HA (2016) Proactive, but not reactive, distractor filtering relies on local modulation of alpha oscillatory activity. J Cogn Neurosci 28:1964-1979.

Vogel EK, McCollough AW, Machizawa MG (2005) Neural measures reveal individual differences in controlling access to working memory. Nature 438:500-503.

Walsh BJ, Buonocore MH, Carter CS, Mangun GR (2011) Integrating conflict detection and attentional control mechanisms. J Cogn Neurosci 23:2211-2221.

Wang B, Theeuwes J (2018a) How to inhibit a distractor location? statistical learning versus active, top-down suppression. Atten Percept Psychophys $80: 860-870$.

Wang B, Theeuwes J (2018b) Statistical regularities modulate attentional capture. J Exp Psychol Hum Percept Perform 44:13-17.

Wang C, Rajagovindan R, Han SM, Ding M (2016) Top-down control of visual alpha oscillations: sources of control signals and their mechanisms of action. Front Hum Neurosci 10:15.

Weiss M, Lavidor M (2012) When less is more: evidence for a facilitative cathodal tDCS effect in attentional abilities. J Cogn Neurosci 24:18261833.

Weissman DH, Roberts KC, Visscher KM, Woldorff MG (2006) The neural bases of momentary lapses in attention. Nat Neurosci 9:971-978.

White BJ, Kan JY, Levy R, Itti L, Munoz DP (2017) Superior colliculus encodes visual saliency before the primary visual cortex. Proc Natl Acad Sci U S A 114:9451-9456.

Yantis S, Jonides J (1990) Abrupt visual onsets and selective attention: voluntary versus automatic allocation. J Exp Psychol Hum Percept Perform $16: 121-134$.

Zhou W, Shu H (2017) A meta-analysis of functional magnetic resonance imaging studies of eye movements and visual word reading. Brain Behav 7:e00683.

Zmigrod S, Zmigrod L, Hommel B (2016) Transcranial direct current stimulation (tDCS) over the right dorsolateral prefrontal cortex affects stimulus conflict but not response conflict. Neuroscience 322:320 325. 